\title{
What determines the cost-effectiveness of diabetes screening?
}

Received: 31 August 2005 / Accepted: 28 February 2006 / Published online: 26 April 2006

C) Springer-Verlag 2006

\begin{abstract}
Aims/hypothesis: The cost-effectiveness of screening for diabetes is unknown but has been modelled previously. None of these models has taken account of uncertainty. We aimed to describe these uncertainties in a model where the outcome was CHD risk. Subjects and methods: Our model used population data from the Danish Inter99 study, and simulations were run in a theoretical population of $1,000,000$ individuals. CHD risk was estimated using the UK Prospective Diabetes Study (UKPDS) risk engine, and risk reduction from published randomised clinical trials. Probabilistic sensitivity analysis was used to provide confidence intervals for modelled outputs. Uncertain parameter values were independently simulated from distributions derived from existing literature and deterministic sensitivity analysis performed using
\end{abstract}

C. Glümer · M. Yuyun · S. Griffin · N. J. Wareham $(\bowtie)$

Medical Research Council Epidemiology Unit,

Elsie Widdowson Laboratories,

120 Fulbourn Road,

Cambridge, CB1 9NL, UK

e-mail: nick.wareham@mrc-epid.cam.ac.uk

Tel.: +44-1223-330315

Fax: +44-1223-330316

C. Glümer

Steno Diabetes Center,

Gentofte, Denmark

C. Glümer

Research Center for Prevention and Health,

Glostrup, Denmark

D. Farewell

Department of Mathematics and Statistics,

University of Lancaster,

Lancaster, UK

D. Spiegelhalter

Medical Research Council Biostatistics Unit,

Cambridge, UK

\section{A. L. Kinmonth}

General Practice and Primary Care Research Unit,

University of Cambridge,

Cambridge, UK multiple model runs under different strategy choices and using extreme parameter estimates. Results: In the least conservative model (low costs and multiplicative risk reduction for combined treatments), the $95 \%$ confidence interval of the incremental cost-effectiveness ratio varied from $£ 23,300-82,000$. The major contributors to this uncertainty were treatment risk reduction model parameters: the risk reduction for hypertension treatment and UKPDS risk model intercept. Overall cost-effectiveness ratio was not sensitive to decisions about which groups to screen, nor the costs of screening or treatment. It was strongly affected by assumptions about how treatments combine to reduce risk. Conclusions/interpretation: Our model suggests that there is considerable uncertainty about whether or not screening for diabetes would be costeffective. The most important but uncertain parameter is the effect of treatment. In addition to directly influencing current policy decisions, health care modelling can identify important unknown or uncertain parameters that may be the target of future research.

Keywords Cost-effectiveness · Decision-modelling · Diabetes $\cdot$ Screening $\cdot$ Uncertainty

Abbreviations ADDITION: Anglo-Danish-Dutch Study of Intensive Treatment in People with Screen Detected Diabetes in Primary Care - CVD: cardiovascular disease - ICER: incremental cost-effectiveness ratio - NNS: number needed to screen - NNT: number needed to treat . QALY: quality-adjusted life-year · UKPDS: UK Prospective Diabetes Study · WHO: World Health Organization

\section{Introduction}

Whether or not screening for undiagnosed type 2 diabetes in asymptomatic individuals is cost-effective is unknown, and policy statements on this topic are controversial. To some it is self-evident that screening should be commenced, given the rapidly increasing prevalence of type 2 
diabetes [1], the fact that around 50\% of individuals with type 2 diabetes are undiagnosed [1], that $20-30 \%$ have complications at clinical diagnosis, and that individuals with type 2 diabetes have a two- to four-fold increased risk of dying from cardiovascular disease (CVD) [2]. To others the evidence that screening is an appropriate public policy is less clear cut, and recommendations focus on screening high-risk groups rather than the whole population $[3,4]$.

In the absence of definitive trial evidence of the net benefits of a programme of screening and subsequent treatment, modelling is an appropriate alternative approach to inform public health strategy. This has previously been undertaken for the effects of screening on the microvascular complications of diabetes [5], but there are few models focusing on macrovascular disease [6], which is, by far, the greater public health problem associated with diabetes. Furthermore, no studies have modelled the combined effect of treatment of hyperglycaemia, hypertension and dyslipidaemia in people with screen-detected diabetes. In this paper we report initial results from a model of screening, with an emphasis on identifying the parameters within the model that have the greatest impact on the estimates of cost-effectiveness. This use of modelling can inform decisions about priorities for research as it can identify important but poorly estimated parameters that can be more accurately quantified in future studies.

\section{Subjects and methods}

The model

Figure 1 outlines the components of the model to estimate the costs and benefits of screening and treatment for type 2 diabetes, with benefit expressed in terms of CHD events prevented over 5 years. Two classes of inputs have been specified: those that concern underlying parameters that predict screening compliance, baseline risk, treatment risk reduction and cost, and choices concerning screening and treatment strategies. For a range of parameter assumptions and strategy choices, the model calculates predicted outcomes for each individual, and these are then weighted to estimate the expected costs and benefits for the chosen strategy in the proposed screening target population. This method of sampling using individual level information from a population-based study avoids the need to build statistical models for population characteristics from which theoretical individuals are simulated.

Parameter assumptions Table 1 shows the assumptions used in the model. For uncertain quantities, we distinguish between those for which an evidence-based probability distribution can be derived from the literature (probabilistic sensitivity analysis), and those that are taken as 'unknown'; in principle, a judgemental distribution could be used for the latter, but instead we have considered a range of informed possible values (deterministic sensitivity analysis) [7]. The overall compliance with a screening programme for type 2 diabetes is unknown. In this paper, sensitivity analyses with levels of compliance ranging from $30-75 \%$ were performed $[8,9]$.The untreated risk model uses the risk prediction engine derived from the UK Prospective Diabetes Study (UKPDS), calculating the 5-year cumulative risk of CHD events (fatal or non-fatal myocardial infarction or sudden death) in people with diabetes (Table 1) [10]. The relative risk reduction for treatment of individual cardiovascular risk factors used in the risk reduction model are taken from a meta-analysis of single agent clinical trials in people with diabetes [11]. By using these clinical trial risk reduction estimates, we are implicitly assuming that the adherence to medication among patients in a real-life screening programme would be identical to that seen in the ideal situation of a randomised controlled trial. None of these trials was
Fig. 1 Cost and effectiveness model of screening and treatment strategies for type 2 diabetes

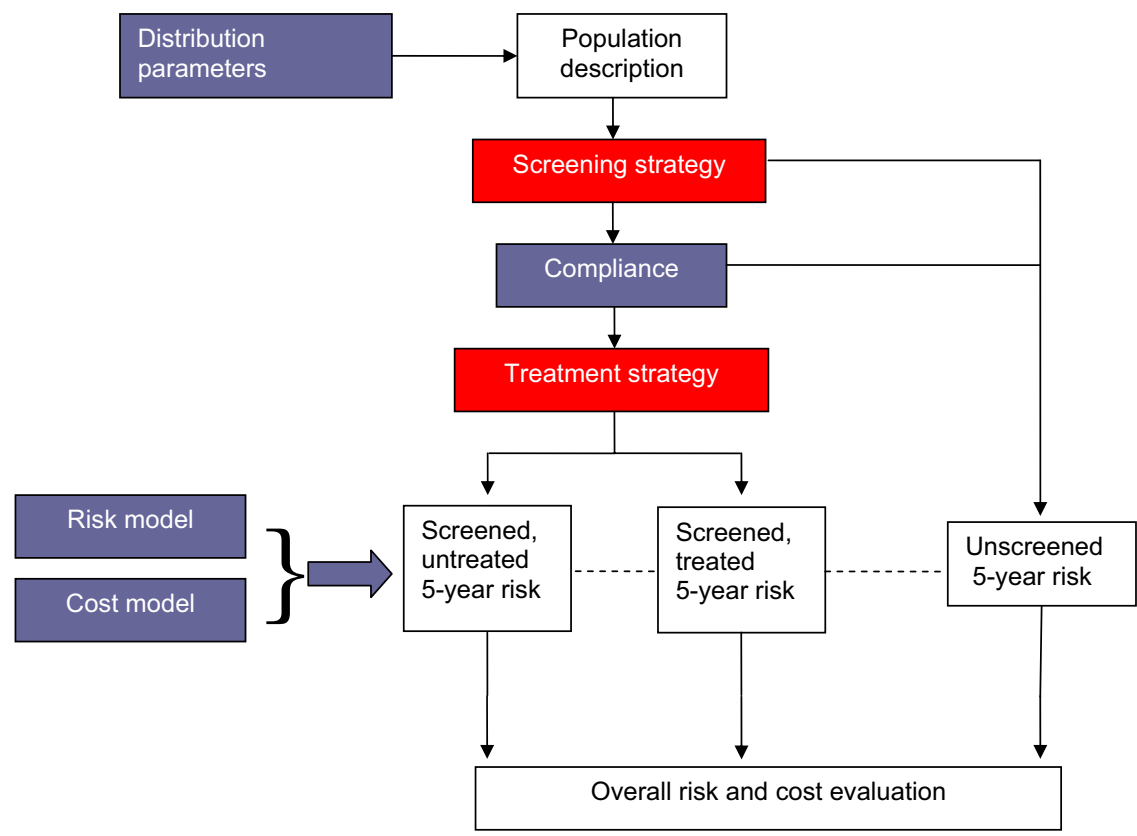


specifically undertaken among individuals with screendetected diabetes, and therefore we assume that risk reductions observed in the populations recruited to these clinical trials can be generalised to people whose diabetes is detected through screening. Most trials including people with diabetes have focused on the management of individual cardiovascular risk factors. Consequently, there is uncertainty concerning the $\mathrm{CHD}$ event rate reduction consequent upon intensive treatment for several cardiovascular risk factors at once. Given that in the real world of current diabetes care most patients with screen-detected diabetes have more than one cardiovascular risk factor, polypharmacy is the norm rather than the exception. Thus, modelling the effects on the results of screening of different assumptions about how single cardiovascular risk factor reductions combine when more than one factor is treated is an important component of this study. We have included two extreme scenarios in our model: the conservative situation, where combined therapy is only as effective as the most effective single agent, and the optimistic scenario, where risk reductions combine in a multiplicative manner (Table 1). The cost model contains information on the cost of the screening procedure estimated from our experience with the ongoing Anglo-

Table 1 Assumptions in the cost-effectiveness model

\begin{tabular}{|c|c|c|}
\hline Parameter & Estimate & $95 \% \mathrm{CI}$ \\
\hline \multicolumn{3}{|l|}{ Compliance: } \\
\hline Worst scenario & $30 \%$ & \\
\hline Best scenario & $75 \%$ & \\
\hline \multicolumn{3}{|l|}{ Risk model: 5 years risk of coronary heart disease: } \\
\hline Intercept & 0.0112 & $0.0082-0.014$ \\
\hline Risk ratio 1 year of age at diagnosis of diabetes & 1.059 & $1.05-1.07$ \\
\hline Risk ratio for female sex & 0.525 & $0.42-0.63$ \\
\hline Risk ratio for Afro-Caribbean ethnicity & 0.390 & $0.19-0.59$ \\
\hline Risk ratio for smoking & 1.350 & $1.11-1.59$ \\
\hline Risk ratio for $1 \%$ increase in $\mathrm{HbA}_{1 \mathrm{c}}$ & 1.183 & $1.11-1.25$ \\
\hline Risk ratio for $10 \mathrm{mmHg}$ increase in systolic blood pressure & 1.088 & $1.04-1.14$ \\
\hline Risk ratio for unit increase in logarithm of lipid ratio & 3.845 & $2.59-5.10$ \\
\hline Risk ratio for each year increase in duration of diagnosed diabetes & 1.078 & $1.05-1.11$ \\
\hline \multicolumn{3}{|l|}{ Risk reduction due to medical treatment: } \\
\hline \multicolumn{3}{|l|}{ Monotherapy } \\
\hline Glucose-lowering treatment & 0.87 & $0.74-1.01$ \\
\hline Blood pressure-lowering treatment & 0.73 & $0.57-0.94$ \\
\hline Lipid-lowering treatment & 0.75 & $0.61-0.93$ \\
\hline \multicolumn{3}{|l|}{ Polytherapy: no additional effect } \\
\hline Glucose- + blood pressure-lowering treatment & 0.73 & $0.74-1.01$ \\
\hline Glucose- + lipid-lowering treatment & 0.75 & $0.57-0.94$ \\
\hline Glucose- + blood pressure- + lipid-lowering treatment & 0.73 & $0.61-0.93$ \\
\hline \multicolumn{3}{|l|}{ Polytherapy: multiplicative effect } \\
\hline Glucose- + blood pressure-lowering treatment & 0.64 & $0.46-0.82$ \\
\hline Glucose- + lipid-lowering treatment & 0.65 & $0.49-0.82$ \\
\hline Glucose- + blood pressure- + lipid-lowering treatment & 0.48 & $0.32-0.66$ \\
\hline \multicolumn{3}{|l|}{ Cost model: } \\
\hline \multicolumn{3}{|l|}{ Current cost, cost per annum per individual (£) } \\
\hline Screening procedure & 10 & \\
\hline Sulfonylurea & 81 & \\
\hline Metformin & 26.4 & \\
\hline Blood pressure control & 156 & \\
\hline Statin & 240 & \\
\hline Service cost & 86 & \\
\hline \multicolumn{3}{|l|}{ Low cost, cost per annum per individual ( $£$ ) } \\
\hline Screening procedure & 10 & \\
\hline Sulfonylurea & 81 & \\
\hline Metformin & 26.4 & \\
\hline Blood pressure control & 117 & \\
\hline Statin & 180 & \\
\hline Service cost & 86 & \\
\hline
\end{tabular}


Danish-Dutch Study of Intensive Treatment in People with Screen Detected Diabetes in Primary Care (ADDITION) [12]. We took the costs of pharmaceutical treatment of hyperglycaemia, hypertension and dyslipidaemia from the British National Formulary [13]. The costs of GP and practice nurse visits were estimated using standard BMA or Whitley Council rates and the frequency of consultations from the UKPDS [14]. The cost of specific pharmaceutical treatments is not fixed and may vary significantly, especially when patents on certain agents cease. Thus, in our sensitivity analyses we estimated the costs and benefits of an alternative situation in which there was a $25 \%$ reduction in cost of treatment with antihypertensive drugs and statins (Table 1).

Screening and treatment strategy choices In the model, it is possible to examine different screening strategies according to information on age, BMI, sex and ethnicity. In this paper, a range of high-risk strategies are evaluated. It is also possible to specify alternative treatment strategies. In the current model, the individual's BMI is used to decide whether they are prescribed a sulfonylurea or metformin; those with a BMI $>25 \mathrm{~kg} / \mathrm{m}^{2}$ being prescribed metformin. The decision to treat with antihypertensive drugs is conditional on the blood pressure, with therapy being commenced if the systolic blood pressure is $\geq 140 \mathrm{mmHg}$ or the diastolic blood pressure is $\geq 85 \mathrm{mmHg}$. Finally, we have assumed that treatment with statins will be commenced for primary prevention of CHD on the basis of an estimated 30\% 10-year Framingham CHD risk [15].

Outcomes For each set of parameter assumptions and strategy choices, the model estimates the proportion of the total population offered screening, the ratio of those screened who subsequently require treatment, the total cost of the screening programme for a population of $1,000,000$ individuals aged $35-60$ years, the numbers needed to screen and treat over 5 years to prevent one CHD event, the total number of CHD events prevented, and the cost-effectiveness ratio (defined as the total cost divided by the number of events prevented). Compared with a baseline strategy of no screening, at no cost and saving no events, these cost-effectiveness ratios can be interpreted as incremental cost-effectiveness ratios (ICERs) [7].

\section{Study population and survey procedures}

In this paper we have used a population-based study from Denmark as an example. The model can be readily tested on data from other population-based studies. The Danish Inter99 study is a primary prevention study of CVD, impaired glucose tolerance and type 2 diabetes in the western part of the Copenhagen County of Denmark [16, 17]. A random sample of 13,016 people aged $30-60$ years, stratified by age and sex, were invited for a health examination including a standard 75-g OGTT. The participation rate was $52.5 \%(n=6,784)$. All individuals with known diabetes $(n=139)$, and 374 people who could not be categorised according to the World Health Organization (WHO) criteria owing to lack of plasma glucose measurements were excluded. Only people with complete information on ethnicity, smoking, sex, age, blood pressure, BMI and blood tests were included in the analyses $(n=6,216$; 93.5\%). The mean of two measurements of blood pressure, taken with the participant lying, using a standard mercury sphygmomanometer with an appropriate cuff size after at least $5 \mathrm{~min}$ rest, was used in the analysis. Weight and height were measured with the participants wearing indoor clothes without shoes. BMI was defined as weight in kilograms divided by height in metres squared. In Denmark, $51 \%$ of the adult population aged 35-80 years are men and $97 \%$ of this population are Europids (Statistics Denmark. Available at http://www.dst.dk./, last accessed 10th August 2005). Plasma glucose was analysed using the hexokinase/G6PDH method (Boehringer Mannheim, Mannheim, Germany). $\mathrm{HbA}_{1 \mathrm{c}}$ samples in capillary tubes were analysed by ion exchange HPLC (Bio-Rad Variant, Richmond, CA, USA). Serum cholesterol was determined using enzymatic techniques (Boehringer Mannheim). Diabetes was defined according to the WHO criteria set out in 1999 [18]. All participants gave written consent before taking part in the study. The protocol was conducted in accordance with the Declaration of Helsinki and approved by the local research ethics committee.

\section{Statistical analysis}

Sensitivity analysis Deterministic sensitivity analysis resulted from multiple runs of the model under different strategy choices and using the extremes of the ranges for the parameters shown in Table 1. Probabilistic sensitivity analysis provided confidence intervals around the outputs from the model using the following method: uncertain parameter values were independently simulated from the distributions in Table 1, the results for each simulated set were recorded, and the subsequent distribution of outcomes were summarised by a mean and $95 \%$ intervals [7]. Sufficient simulations were carried out to estimate the intervals with under 5\% error. All modelling was carried out in Excel 2000 (Microsoft, Redmond, WA, USA), with the addition of Crystal Ball http://www.decisioneering. com/crystal_ball, last accessed in February 2006) for the probabilistic sensitivity analysis.

Other statistical analyses were carried out using SAS, version 8.2 (SAS institute, Cary, NC, USA). Groups were compared using the Student's $t$-test for continuous variables, the $\chi^{2}$ test for dichotomous variables. A $p$ value of $<0.05$ was considered statistically significant.

\section{Results}

In the Inter99 study, the prevalence of screen-detected diabetes was $4.3 \%$ (95\% CI 3.9-4.9\%). The characteristics 
of the Inter99 population are shown in Table 2. Individuals with diabetes had significantly higher $\mathrm{HbA}_{1 \mathrm{c}}$, blood pressure, and cholesterol:HDL cholesterol ratios, and were more likely to be men compared with individuals without diabetes. Hypertension was present in $75.6 \%$ of individuals with diabetes compared with $35.4 \%$ of individuals without diabetes. These findings demonstrate the higher prevalence of cardiovascular risk factors in individuals with screendetected diabetes compared with those without diabetes. There were no differences between the two groups in terms of smoking status or ethnicity.

Table 3 shows the results for different theoretical highrisk screening strategies in a population of $1,000,000$ individuals aged $30-60$ years. It assumes a response rate for screening of $75 \%$, a multiplicative risk from multiple therapy, and a cost model using current costs. The total cost of the evaluated screening strategies varied between $£ 13.3$ and $£ 27.4$ million. In addition, the number needed to screen (NNS) varied between 240 and 362, and the number needed to treat (NNT) varied between 32 and 34 . The NNS, NNT, and cost-effectiveness ratio did not change within the different strategies, but the total cost of the entire programme varied markedly according to strategy. The high-risk strategies were cheaper than the broader strategies. However, the number of events prevented was lowest in the high-risk strategies, and highest in the mass strategies.

Sensitivity analyses are given in Table 4 for the fixed strategy of screening all those aged $\geq 50$ years with a BMI $>30 \mathrm{~kg} / \mathrm{m}^{2}$. The models refer to the different deterministic sensitivity analyses, with stepwise changes in the parameter assumptions as in Table 1. In the models assuming a multiplicative effect on risk reduction according to treatment, the number of coronary heart disease events prevented was higher than in the models assuming no additional effect. Consequently, the NNS, NNT and ICER were all lower in the multiplicative models.

Table 2 Characteristics of the Inter99 population

\begin{tabular}{lll}
\hline & No diabetes & $\begin{array}{l}\text { Screen-detected } \\
\text { diabetes }\end{array}$ \\
\hline Number & 5956 & 260 \\
Men (\%) & $49.2(2928)$ & $63.5(165)$ \\
Non-Europid (\%) & $1.5(92)$ & $1.5(4)$ \\
Age (years) & $45.8 \pm 7.9$ & $50.5 \pm 6.9$ \\
BMI (kg/m $\left.{ }^{2}\right)$ & $26.1 \pm 4.4$ & $30.1 \pm 5.8$ \\
HbA 1 (\%) & $5.8 \pm 0.4$ & $6.6 \pm 1.4$ \\
Systolic blood pressure (mmHg) & $129.4 \pm 16.7$ & $146.3 \pm 20.5$ \\
Diastolic blood pressure (mmHg) & $82.0 \pm 11.1$ & $90.6 \pm 11.8$ \\
Cholesterol:HDL ratio & $4.1 \pm 1.4$ & $5.1 \pm 1.6$ \\
Current smokers (\%) & $39.7(2367)$ & $39.6(103)$ \\
Hypertensive (\%) & $35.4(2106)$ & $74.6(194)$ \\
UKPDS predicted 5-year cumulative & - & $6.4 \pm 5.4$ \\
risk of having a CHD event (\%) & & \\
\hline
\end{tabular}

Values are means \pm SD or percent and (numbers)

Individuals with no diabetes included individuals with NGT, IFG or IGT

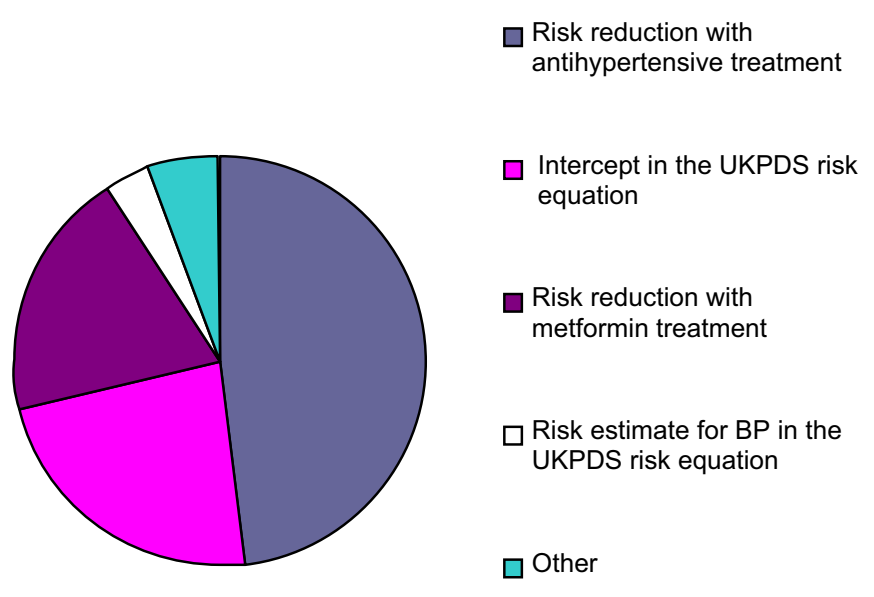

Fig. 2 The contribution to the variance of the sensitivity analysis

Decreasing the compliance reduced the number of events prevented but did not affect the NNT, NNS or ICER. Changing the cost by $25 \%$ for the treatment of hypertension and dyslipidaemia affected the ICER but not the NNS or NNT.

The uncertainties of the estimates simulated by probabilistic sensitivity analysis are given as $95 \%$ prediction limits. For all outcome estimates there was a five-fold increase from the lowest to the highest limit. The impact on the variance from the probabilistic sensitivity analysis of the model is shown in Fig. 2. The uncertainty of the risk reduction associated with antihypertensive treatment contributed most to the variance of the model (48\%), followed by the intercept (corresponding to the baseline hazard for a 55-year-old, non-smoking Europid or Asian-Indian man with a $\mathrm{HbA}_{1 \mathrm{c}}$ of $6.72 \%$, a systolic $\mathrm{BP}$ of 135.7 and a lipid ratio of 4.9 ) in the UKPDS risk engine (23\%), and then treatment with hypoglycaemic medication $(20 \%)$.

\section{Discussion}

In an ideal world, policy decisions about whether or not to screen for conditions like type 2 diabetes would be made on the basis of perfect information with a strong evidence base supported by randomised controlled trials, not only of the specific treatments but of the whole process of screening. However, this is far from reality, as such public health policy considerations are often driven by timetables that do not allow for long-term trials, and there may be objections to trials of the process of screening. In the absence of these definitive data, modelling becomes a critical public policy tool. A key aspect of modelling that is often overlooked is uncertainty, since for many of the parameters that are included in these models there is either no published evidence at all, or else only estimations available. In this paper we have described the development of a simple simulation model for estimating the cost-effectiveness of screening for type 2 diabetes. More importantly, we have shown how choosing different high-risk screening ap- 


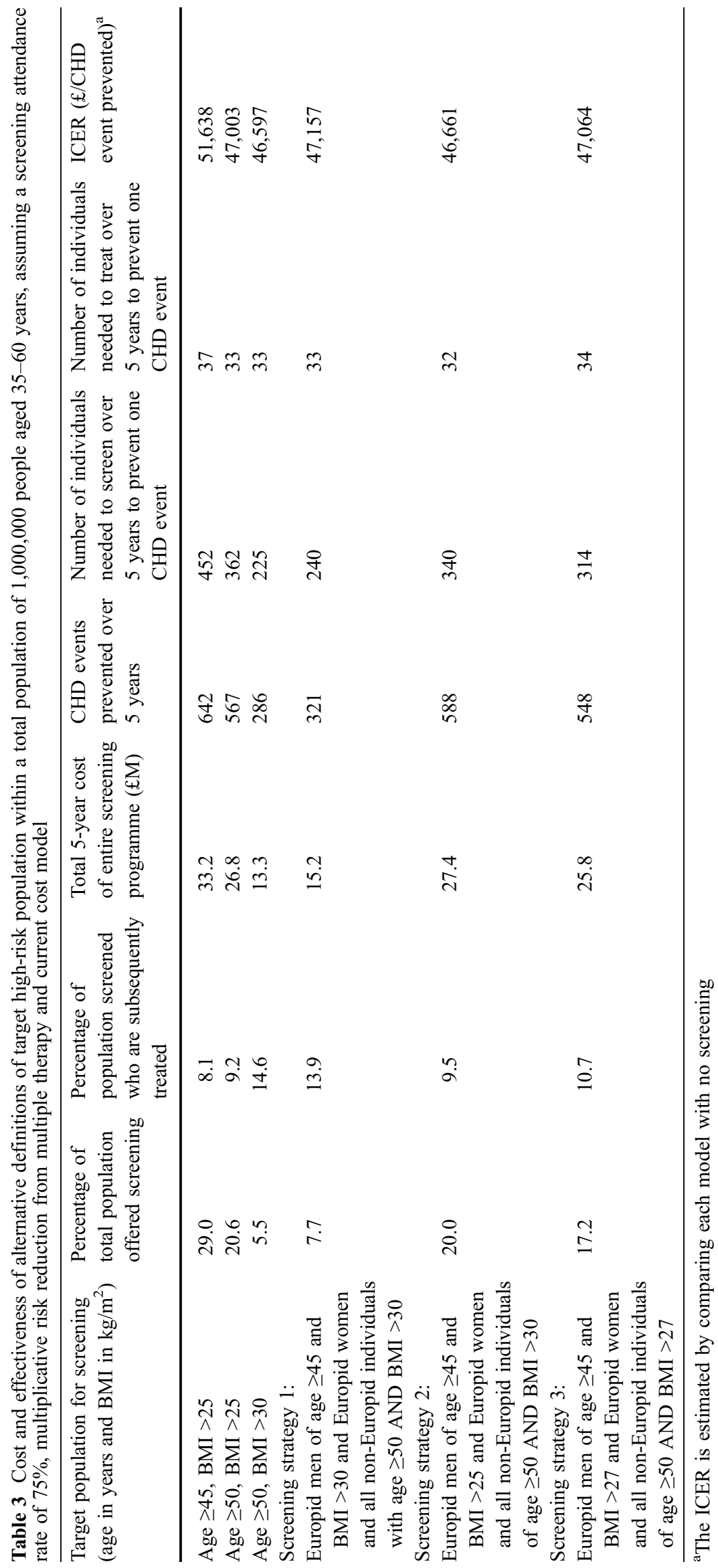


Table 4 Monte Carlo simulations and sensitivity analysis for results of screening varying compliance, assumption of treatment risk reduction and cost model in a screening strategy aimed at individuals aged $\geq 50$ years with a BMI $>30 \mathrm{~kg} / \mathrm{m}^{2}$

\begin{tabular}{llllllllllll}
\hline & $\begin{array}{l}\text { Compliance } \\
(\%)\end{array}$ & $\begin{array}{l}\text { Treatment risk } \\
\text { reduction model }\end{array}$ & $\begin{array}{l}\text { Cost } \\
\text { model }\end{array}$ & $\begin{array}{l}\text { CHD events } \\
\text { prevented } \\
\text { over 5 years }\end{array}$ & NNS & NNT & ICER \\
\hline Model 1 & 30 & No additional effect & Low & 77 & $27-140$ & 333 & $183-938$ & 49 & $27-137$ & 59,500 & $32,700-167,300$ \\
Model 2 & 30 & No additional effect & Current & 77 & $27-140$ & 334 & $183-938$ & 49 & $27-137$ & 69,100 & $38,000-194,300$ \\
Model 3 & 75 & No additional effect & Low & 192 & $68-351$ & 334 & $183-938$ & 49 & $27-138$ & 59,500 & $32,700-167,300$ \\
Model 4 & 75 & No additional effect & Current & 192 & $66-351$ & 336 & $183-958$ & 49 & $27-140$ & 69,500 & $37,800-198,400$ \\
Model 5 & 30 & Multiplicative effect & Low & 113 & $57-181$ & 227 & $143-450$ & 33 & $21-66$ & 40,500 & $25,400-80,200$ \\
Model 6 & 30 & Multiplicative effect & Current & 113 & $57-179$ & 228 & $143-453$ & 34 & $21-66$ & 47,300 & $29,800-94,000$ \\
Model 7 & 75 & Multiplicative effect & Low & 282 & $140-454$ & 228 & $142-460$ & 33 & $21-67$ & 40,700 & $23,300-82,000$ \\
Model 8 & 75 & Multiplicative effect & Current & 284 & $145-462$ & 227 & $139-444$ & 33 & $20-65$ & 46,900 & $28,800-92,000$ \\
\hline
\end{tabular}

This table shows the deterministic sensitivity analysis in the left side of the table varying the assumptions of compliance, treatment risk reduction and cost one by one. The numbers are the medians and the $95 \%$ prediction limits of the medians after running a Monte Carlo simulation

proaches with various permutations of target groups based on age, degree of obesity, and ethnicity made no significant difference to the number of individuals needed to be screened or treated to prevent one CHD event, or to the cost-effectiveness ratio. However, the major impact of these different policy decisions was on the total cost of the screening programme and the total number of CHD cases prevented, with both increasing as strategies were broadened to include a greater proportion of the population.

A major advantage of this model is that it incorporates the risk of developing CHD and models the effect of simultaneous treatment of hyperglycaemia, hypertension and dyslipidaemia. Previous modelling studies have tended to focus on the efficiency of case detection or have restricted benefits to the effect of treatment of hyperglycaemia $[5,19-21]$ or the effect of combined treatment of hyperglycaemia and hypertension [6]. A final key feature of this analysis was the focus on uncertainty. All screening models contain uncertain factors, some of which can be estimated from the literature, and others that can only be taken as 'unknown' and a range of plausible values assessed. No previous models have explored the impact of this uncertainty on the results. Our results suggest that decisions about whether or not to screen for diabetes using such models now would be based on data that has considerable uncertainty. The overall cost-effectiveness ratio does not appear to be sensitive to decisions about which groups to screen, or particularly to the costs of screening or treatment. It is, however, affected to a large degree by the assumptions about how treatments combine to reduce risk. It is not currently known whether the risk reduction associated with treating hypertension, dyslipidaemia and hyperglycaemia in people with screen-detected diabetes are multiplicative, additive or sub-additive. Data from studies such as the Steno 2 trial, in which multifactorial therapy was compared with standard care, can provide some evidence of how risk is reduced by combined treatment, but this is limited since the trial was conducted in high-risk individuals, i.e. people with established diabetes and microalbuminuria [22]. The impact of this type of un- certainty on the estimates of cost-effectiveness provides justification for trials such as ADDITION, which aims to measure the effect of intensified multifactorial therapy on CHD events in people with screen-detected diabetes [12].

In addition to the impact of unknown parameters, such as the combined treatment risk reduction, we also investigated the uncertainty in the estimates of cost-effectiveness attributable to the evidence-based uncertainties in critical parameters. In our least conservative model, where costs were low and the impact of combined treatments was multiplicative, the $95 \%$ confidence interval of the ICER varied from $£ 23,300-82,000$. The major contributors to this uncertainty were the parameters in the treatment risk reduction model - particularly the risk reduction from treatment of hypertension in people with diabetes - and the intercept of the UKPDS risk model. Models like this can help to direct research efforts to narrow confidence intervals in key but uncertain parameters.

In our model we have only focused on CHD events. Others have modelled the effects of screening on microvascular disease. The Centers for Disease Control and Prevention (CDC) group found that universal screening for type 2 diabetes was not cost-effective in the age range in which it is most prevalent - those people aged 55-64 years -in whom the cost per quality-adjusted life-year (QALY) was US\$70,759 and the cost per life-year gained was US $\$ 480,600$. In the $>65$ years age group the cost per QALY was US\$575,000 [19]. The ICER was lower in younger individuals. In light of the already established uncertainty about the benefits of screening for diabetes on microvascular outcomes, and the much greater public health burden of undiagnosed diabetes attributable to CHD risk, our model focused on this outcome. It is likely however, that reductions in CHD mortality attributable to early, more intensive multifactorial therapy would also have effects on microvascular outcomes, which might, in turn, alter the balance between the costs and benefits of screening over the longer term. Thus, future iterations of this model will need to consider multiple health endpoints. The time horizon for this analysis was 5 years, a time-course over 
which the issues of discounting are not brought to the fore. However, if a longer time perspective were taken, then the model would also need to be complicated by consideration of discounting. Finally, we consider screening strategies based on selection of population groups based on easily identifiable characteristics, such as age, ethnicity and degree of obesity. Future developments of our model could include consideration of other strategies, although the contribution to the outcome of cost-effectiveness would be unlikely to be major, since we have already shown that the screening strategy has a limited impact on the ICER, although it does have an impact on the total cost of the programme.

This kind of model does have other limitations. We focused our attention on treatments for hyperglycaemia, hypertension and dyslipidaemia. Other treatments, such as antiplatelet therapy including acetylsalicylic acid (ASA) could have been included, since some, but not all, expert guidelines recommend cardioprotective treatment with ASA $[23,24]$. However, there is no clear evidence that ASA treatment is effective in primary prevention in individuals with diabetes $[25,26]$, as a meta-analysis from 2002 that included 5,955 individuals with diabetes reported that treatment with ASA was associated with a non-significant $7 \%$ reduction in the risk of having any vascular event [26]. Using the UKPDS risk engine may overestimate the risk of CHD since it was developed in trial participants, the majority of whom had clinically diagnosed diabetes and are therefore likely to have had disease for longer than screen-detected cases, with greater possibility for accumulation of time-dependent vessel damage. In the treatment model, metformin was initiated without reference to $\mathrm{HbA}_{1 \mathrm{c}}$. This is an assumption that increases potential benefit but has little effect on cost because the drug is cheap. In reality, many of the screen-detected patients would actually not receive any specific hypoglycaemic medication at the point of detection by screening, as $57 \%$ have a $\mathrm{HbA}_{1 \mathrm{c}}<6.5 \%$. Over time, many of these patients would have therapy added as $\mathrm{HbA}_{1 \mathrm{c}}$ rises with duration of disease, but our model has assumed treatment from diagnosis. It is unknown whether early hypoglycaemic therapy alters the disease trajectory. The cost model used in this analysis is naïve as we have used average costs. We have not estimated the effects of uncertainty in cost, and the effect of adding in this additional level of uncertainty would only add to the overall uncertainty in the ICER. We have not included strategies for differential targeting of specific ethnic groups, principally because the study population chosen as an example is overwhelmingly Europid. Future work should consider inclusion of other populations in which data from other ethnic groups are available.

In conclusion, we have developed a cost-effectiveness model estimating the benefits of screening for type 2 diabetes. The impact of uncertainty in key parameters demonstrates the importance of including sensitivity analyses in future models and highlights areas where research efforts may narrow uncertainty. The demonstration of the impact of factors that are unknown helps to inform research strategy. Our analysis suggests that measuring the risk reduction from multifactorial therapy in people with screen-detected diabetes is a priority.

Acknowledgements The work of D. Farewell in developing the screening model was funded by the UK Department of Health.

We are grateful to the Steering Committee of the Inter99 study ( $T$. J $\phi$ rgensen, K. Borch-Johnsen. H. Ibsen, T. Thomsen, C. Pisinger and C. Glümer) for allowing us to include their data in the model. We thank the participants in this study, the Inter99 staff and the laboratory at the Steno Diabetes Center. The Inter99 study was supported by the Danish Medical Research Council, the Danish Centre for Evaluation and Health Technology Assessment, Novo Nordisk, Copenhagen County, the Danish Heart Foundation, the Danish Diabetes Association, the Danish Pharmaceutical Association, the Augustinus foundation, the Ib Henriksen Foundation, the Becket Foundation, and Glaxo SmithKline.

\section{References}

1. King H, Aubert RE, Herman WH (1998) Global burden of diabetes, 1995-2025: prevalence, numerical estimates, and projections. Diabetes Care 21:1414-1431

2. Glucose tolerance and mortality: comparison of WHO and American Diabetes Association diagnostic criteria. The DECODE study group. European Diabetes Epidemiology Group. Diabetes Epidemiology: Collaborative analysis Of Diagnostic criteria in Europe. Lancet 1999;354:617-621

3. Borch-Johnsen K, Lauritzen T, Glumer C, Sandbaek A (2003) Screening for Type 2 diabetes - should it be now? Diabet Med 20:175-181

4. Wareham NJ, Griffin SJ (2001) Should we screen for type 2 diabetes? Evaluation against national screening committee criteria. BMJ 322:986-988

5. Hofer TP, Vijan S, Hayward RA (2000) Estimating the microvascular benefits of screening for type 2 diabetes mellitus. Int J Technol Assess Health Care 16:822-833

6. Hoerger TJ, Harris R, Hicks KA, Donahue K, Sorensen S, Engelgau M (2004) Screening for type 2 diabetes mellitus: a cost-effectiveness analysis. Ann Intern Med 140:689-699

7. Briggs AH (2000) Handling uncertainty in cost-effectiveness models. Pharmacoeconomics 17:479-500

8. Lawrence JM, Bennett P, Young A, Robinson AM (2001) Screening for diabetes in general practice: cross sectional population study. BMJ 323:548-551

9. Williams DR, Wareham NJ, Brown DC et al (1995) Undiagnosed glucose intolerance in the community: the Isle of Ely Diabetes Project. Diabetic Med 12:30-35

10. Stevens RJ, Kothari V, Adler AI, Stratton IM, Holman RR (2001) The UKPDS risk engine: a model for the risk of coronary heart disease in Type II diabetes (UKPDS 56). Clin Sci 101:671-679

11. Huang ES, Meigs JB, Singer DE (2001) The effect of interventions to prevent cardiovascular disease in patients with type 2 diabetes mellitus. Am J Med 111:633-642

12. Lauritzen T, Griffin S, Borch-Johnsen K, Wareham NJ, Wolffenbuttel BHR, Rutten G (2000) The ADDITION study: proposed trial of the cost-effectiveness of an intensive multifactorial intervention on morbidity and mortality among people with Type 2 diabetes detected by screening. Int J Obes Relat Metab Disord 24:S6-S11

13. Joint Formulary Committee (2002) British National Formulary. British Medical Association, and Royal Pharmaceutical Society of Great Britain, London

14. Turner RC, Holman RR, Cull CA, Stratton IM, Matthews DR, Frighi $\mathrm{V}$ et al (1998) Intensive blood-glucose control with sulfonylureas or insulin compared with conventional treatment and risk of complications in patients with type-2 diabetes (UKPDS 33). Lancet 352:837-853 
15. Wilson PWF, D'Agostino RB, Levy D, Belanger AM, Silbershatz H, Kannel WB (1998) Prediction of coronary heart disease using risk factor categories. Circulation 97:1837-1847

16. Glumer C, Jorgensen T, Borch-Johnsen K (2003) Prevalences of diabetes and impaired glucose regulation in a danish population: the inter99 study. Diabetes Care 26:2335-2340

17. Jorgensen T, Borch-Johnsen K, Thomsen TF, Ibsen H, Glumer C, Pisinger C (2003) A randomized non-pharmacological intervention study for prevention of ischaemic heart disease: baseline results Inter99 (1). Eur J Cardiovasc Prev Rehabil 10:377-386

18. Definition, diagnosis and classification of diabetes mellitus and its complications. Report of a WHO Consultation. Part 1. Diagnosis and classification of diabetes mellitus. 1999. World Health Organization, Geneva 1999. WHO/NCD/NCS/99.2

19. CDC Diabetes Cost-Effectiveness Study Group, Centers for Disease Control and Prevention (1998) The cost-effectiveness of screening for type 2 diabetes. JAMA 280:1757-1763

20. Chen TH-H, Yen MF, Tung TH (2001) A computer simulation model for cost-effectiveness analysis of mass screening for Type 2 diabetes mellitus. Diabetes Res Clin Pract 54:37-42
21. Eastman RC, Javitt JC, Herman WH et al (1997) Model of complications of NIDDM. II. Analysis of the health benefits and cost-effectiveness of treating NIDDM with the goal of normoglycemia. Diabetes Care 20:735-744

22. Gaede P, Vedel P, Larsen N, Jensen GVH, Parving HH, Pedersen O (2003) Multifactorial intervention and cardiovascular disease in patients with type 2 diabetes. N Engl J Med 348:383-393

23. Type 2-diabetes i almen praksis, en evidensbaseret vejledning. 2004. Dansk Selskab for Almen Medicin

24. American Diabetes Association (2006) Standards of medical care in diabetes-2006. Diabetes Care 29:S4-S42

25. Sacco M, Pellegrini F, Roncaglioni MC, Avanzini F, Tognoni G, Nicolucci A (2003) Primary prevention of cardiovascular events with low-dose aspirin and vitamin $\mathrm{E}$ in type 2 diabetic patients: Results of the Primary Prevention Project (PPP) trial. Diabetes Care 26:3264-3272

26. Antithrombotic Trialists' Collaboration (2002) Collaborative meta-analysis of randomised trials of antiplatelet therapy for prevention of death, myocardial infarction, and stroke in high risk patients. BMJ 324:71-86 